\title{
Treating Nonsuicidal Self-Injury
}

\author{
Anna I. Guerdjikova, PhD, LISW ${ }^{1,2, *}$ \\ Iola S. Gwizdowski ${ }^{3}$ \\ Susan L. McElroy, MD ${ }^{1,2}$ \\ Cheryl McCullumsmith, MD, PhD ${ }^{1}$ \\ Patricia Suppes, $M D, P h D^{3}$
}

\author{
Address \\ ${ }^{*}$ DDepartment of Psychiatry and Behavioral Neuroscience, University of Cincinnati \\ College of Medicine, Cincinnati, $\mathrm{OH}$, USA \\ Email: anna.guerdjikova@lindnercenter.org \\ ${ }^{2}$ Lindner Center of HOPE, 4075 Old Western Row Rd, Mason, OH 45040, USA \\ ${ }^{3}$ Department of Psychiatry and Behavioral Sciences, Stanford University Medical \\ Center, Stanford, CA, USA
}

Published online: 3 October 2014

(C) Springer International Publishing AG 2014

Iola S. Gwizdowski Deceased (27 November 2013)

Keywords Nonsuicidal self-injury - Deliberate self-harm - Self-injurious behavior - Burning - Cutting - Naltrexone . Cognitive behavioral therapy

\section{Opinion statement}

Nonsuicidal self-injury (NSSI) evaluation and treatment are critical for comprehensive patient care. A thorough assessment of nonsuicidal self-injury(NSSI) following DSM-5proposed criteria is critical in understanding and treating NSSI and should be informed based upon past and current self-harm behaviors, including self-injury methods, frequency, severity, and intent, along with a comprehensive suicide risk assessment. After NSSI is diagnosed, patients should be offered proper management of co-occurring psychiatric illnesses, if applicable, enhanced by an $8-12$ week trial of cognitive behavior therapy (CBT) focusing on NSSI. Cognitive strategies to be used include Socratic questioning, keeping thought records, and addressing self-derogatory and distorted beliefs about NSSI. Behavioral strategies include contingency management, behavioral activation, and addressing environmental factors maintaining NSSI. In adolescents, individual CBT work might be supplemented with family-based therapy. Dialectical strategies (acceptance and distress tolerance) and skills training (focus on improving emotion regulation, problemsolving, interpersonal, and communication skills) can be used to enhance CBT, but in some cases DBT can be cost prohibitive. If adequate psychotherapy trials are ineffective, a pharmacotherapy trial of emerging drug therapies for NSSI, e.g. naltrexone, Nacetylcysteine, or topiramate to enhance supportive psychotherapy would be recommended. We would encourage patients to continue effective treatment for at least 6 months and would continuously assess for self-harm urges and behaviors. Psychoeducation and adequate 
treatment of comorbid conditions should be provided on an ongoing basis along with attention to physical self-care and regular exercise.

\section{Introduction}

Nonsuicidal self-injury (NSSI) behaviors are a major public health problem and have been reported by clinicians for centuries [1]. Historically, multiple terms were used to describe NSSI, including "deliberate self-harm (DSH)" ,"parasuicide behavior", "self-injurious behavior (SIB)", "self-mutilation", and "self-wounding", and uses of each of the these terms have included both NSSI and self-injury with suicidal intent [2]. NSSI is described as repeated cutting, burning, hitting, rubbing, or otherwise deliberately inflicting damage to body tissue for nonsocially sanctioned reasons, but not as a suicide attempt, and it does not include body manipulations for body adornment (e.g., tattoos or piercings). As these behaviors are often preceded by emotional distress and followed by subjective relief, NSSI is hypothesized to function as a maladaptive attempt to relieve dysphoric affect that might have an addictive or compulsive quality. The most commonly reported reason for NSSI is "to feel anything at all, even pain"; other reasons include "to try to get a reaction from someone, even if it is negative", "to stop bad feelings" or "to get control of the situation" [3]. The injury is most often inflicted with a sharp object; the resulting cuts bleed and leave a characteristic pattern of scars. Common sites for NSSI are the dorsal side of forearm and anterior area of thighs, but any body part may be a target.

Among clinical inpatient samples, estimated prevalence of NSSI is about $21 \%$ for adults [ 4 ] and up to $40 \%$ in adolescents [5]. In community samples, up to $6 \%$ of adults [ $6 \bullet$ and $35 \%$ of adolescents [7] engage in NSSI. The onset of NSSI is commonly between 12 and 16 years of age $[6 \bullet, 8]$. While NSSI is more prevalent among females in adolescence [9], no gender separation is observed in adult community [10] and clinical [11] samples.

Until recently, unified diagnostic criteria for NSSI did not exist, thus research focused on DSH or SIB did not always differentiate between suicidal and nonsuicidal intent. The Diagnostic and Statistical Manual-5 (DSM5) released in May 2013 [12] provided for the first time a concise definition of NSSI. NSSI is included in the "Conditions for Further Study" section of DSM-5, specifying that while proposed criteria are not intended for clinical use, further research on the topic is encouraged.

NSSI, DSM-5 criteria

A. In the last year, the individual has, on 5 or more days, engaged in intentional self-inflicted damage to the surface of his or her body, of a sort likely to induce bleeding or bruising or pain (e.g., cutting, burning, stabbing, hitting, excessive rubbing), with the expectation that the injury will lead to only minor or moderate physical harm (i.e., there is no suicidal intent). The absence of suicidal intent is either stated by the individual or can be inferred by the individual's repeated engagement in the behaviors that the individual knows, or has learned, it is not likely to result in death.

B. The individual engages in self-injurious behavior with one of more of the following expectations: to obtain relief from a negative feeling or cognitive state; to resolve an interpersonal difficulty; to induce a state of positive feeling

C. The intentional injury is associated with at least two of the following:

1. Negative feelings or thoughts, such as depression, anxiety, tension, anger, generalized distress, or selfcriticism occurring in the period immediately prior to the self-injurious act.

2. Prior to engaging in the act, a period of preoccupation with the intended behavior that is difficult to resist.

3. The urge to engage in self-injury occurs frequently, although it might not be acted upon.

D. The behavior is not socially sanctioned and is not restricted to picking a scab or nail biting

E. The behavior or its consequences cause clinically significant distress or interference in interpersonal, academic, or other important areas of functioning.

F. The behavior does not occur exclusively during states of psychosis, delirium, or intoxication. In individuals 
with a developmental disorder, the behavior is not part of a pattern of repetitive stereotypes. The behavior cannot be accounted for by another mental or medical disorder (i.e., psychotic disorder, pervasive developmental disorder, mental retardation, Lesch-Nyhan Syndrome, stereotyped movement disorder with self-injury, or trichotillomania).

NSSI often co-exists with other psychopathology including mood disorders, personality disorders, and eating disorders[5], and a is a strong predictor for future suicide attempts $[13 \bullet]$.

\section{Treatment of NSSI}

The goal of treatment intervention for NSSI is to eliminate self-injurious behaviors, to help control impulses to self-harm, and to prevent relapse and remission of any co-morbid psychiatric conditions. In many cases, this can be achieved only by thorough diagnosis followed by a combination of psychological and pharmacological interventions.

Assessment of NSSI is critical for understanding and treating NSSI. A comprehensive assessment of NSSI should include information on current and past NSSI behavior (types, methods, locations, precipitating factors, frequency, age of onset, severity, urges to self-injure, hospitalizations), understanding of biopsychosocial risk and protective factors for NSSI, and a thorough evaluation of suicide risk and co-morbid psychiatric disorders. Several instruments are available to assist in an assessment of NSSI, such as the Self-Injurious Thoughts and Behaviors Interview [14], the Suicide Attempt Self-Injury Interview [15], and the Ottawa Self-Injury Inventory [16].

No randomized psychotherapy trials have examined DSM-5-defined NSSI behavior in adolescents or adults, and therapeutic programs specifically targeting NSSI are rare. Most published studies have focused on cognitive behavior and dialectical behavior therapeutic techniques in the treatment of self-harm, with or without suicidal intent.

- Cognitive behavior therapy (CBT) and its numerous variations is the most studied modality in DSH and SIB. A recent systematic review and meta-analysis of 28 studies found a highly significant effect for CBT in reducing self-harm in adults when compared to minimal treatment or treatment as usual (TAU) [17].

- $\quad$ CBT is a psychotherapeutic approach that addresses dysfunctional emotions and maladaptive behaviors by challenging cognitive processes. CBT uses some systematic procedures like continuously monitoring dysfunctional behaviors to increase patients' awareness of the problem and further, to help them replace maladaptive coping skills and cognitions with more adaptive ones. Certain components of CBT found useful in reducing deliberate harm include: increasing helpful beliefs, enhancing mood tolerance, changing behavior by increasing activity and improving problem-solving skills, building interpersonal skills through improving communication and improving social functioning, and incorporating relapse prevention [18]. 
- Manual-Assisted Cognitive Behavioral Therapy (MACT) is a program developed for short-term therapy for adults with DSH, including suicidal behavior and NSSI [19]. This cognitive-oriented and problemfocused program consists of a maximum of six therapeutic sessions and is assisted by a detailed manual. The content of MACT involves problem identification and solving, working through advantages and disadvantages of self-harm behaviors and learning basic cognitive strategies to manage emotions and negative thinking, establishing successful coping skills and relapse prevention. In a controlled trial, the MACT program was tested versus TAU in 480 patients following episodes of DSH. While the proportion of those repeating DSH behaviors in the 12 months of the study showed no statistically significant difference between the MACT group and controls, the authors concluded that clinically meaningful findings like $50 \%$ reduction of frequency of selfharm behaviors taken in conjunction with the economic evaluation indicated superiority of MACT over TAU in terms of cost and effectiveness combined [20]. Taylor and colleagues adapted MCAT for adolescents and developed a manualized 12-week CBT individual therapy program ("Cutting Down") including a thorough cognitivebehavioral assessment and systematic learning of motivational strategies and daily coping skills [21]. The 25 adolescents in the pilot study, which was not controlled, reported reduced NSSI after treatment, with continued reduction at a 3-month follow-up.

- Dialectical Behavioral Therapy (DBT) was developed for the treatment of patients with borderline personality disorder (BPD), with a primary focus on self-harm reduction. DBT is multi-pronged approach consisting of skills-based training, individual psychotherapy, telephone calls, and consultation. DBT focus is on teaching the patient how to manage emotional distress through developing interpersonal skills, reality acceptance skills, and mastering emotional regulation. DBT and its shorter treatment duration modification (DBT-Brief) [22] have been shown to reduce effectively NSSI in adults and adolescents across various study designs, most commonly in BPD patients [23]. Modification of DBT for adolescents (DBT-A) has been examined in a study of 12 adolescents with NSSI and other BPD symptoms, and results indicated that DBT-A was helpful in reducing both NSSI and other symptoms of BPD with continued gains at 1-year follow-up [24]. This treatment modality requires highly skilled and trained therapists, and the duration and cost of treatment might be, in some cases, prohibitive for common use.

- Mentalization-based treatment (MBT) has shown to be effective in reducing self-harm in adults [25] and adolescents (MBT-A) [26] with BPD in randomized controlled trials. MBT-A is a year-long, manualized, psychodynamic psychotherapy program, involving weekly, 50-min individual and monthly mentalization-based family sessions with a focus on reducing impulsivity and enhancing affect regulation. Mentalization is conceptualized as the capacity to understand actions in terms of thoughts and feelings. The ability to understand 
one's mental state and its enhancement has been proposed to increase self-control in individuals with affect dysregulation and impulse control problems. In the only randomized controlled trail in MBT of adolescents published to date, a recovery rate of $44 \%$ of the MBT group compared to $17 \%$ in the group receiving TAU was achieved [26]. The authors concluded that "positive change in mentalizing and improvement in interpersonal functioning seem to be the mediating factors in reduction in self-harm".

- Problem-Solving Therapy (PST) is an individual therapy-based model, examining a person's approach to problems, including problem recognition and definition, brainstorming, and devising and continuous reviewing of the action plan. Within a PST model, NSSI is conceptualized as a dysfunctional solution to problems where improved problemsolving skills and increased problem awareness would lead to decreased reliance on NSSI as a coping mechanism. In a randomized trial of 1,094 adult patients admitted to inpatient hospitals with self-harm, only patients treated with PST who had history of SIB prior to current hospitalization were less likely to present again with SIB [27].

- Harm reduction has not been tested in controlled studies but various strategies have been anecdotally reported as potentially helpful in NSSI. A main focus of treatment is seeking to understand the function NSSI serves for the patient and the interventions might include meditation, tapping into spirituality, and increasing patients' self-understanding [28].

- Increased physical activity. Preliminary evidence suggests that regular exercise or participation in sports might hold promise in decreasing urges to engage in NSSI in adolescents [29].

Pharmacotherapy

Dysregulation in endogenous opioids, monoamines, and glutamate neurotransmitter systems are some of the neurobiological mechanisms suggested to be involved in the etiology of NSSI and its maintenance. While there is no Food and Drug Administration (FDA) approved treatment for NSSI, a number of agents modulating the three above-mentioned pathways have been explored. Of note, most of the trials discussed below were performed in individuals with developmental disabilities and this limitation should be taken in consideration when NSSI treatment plan is designed.

\section{Opioid systems modulating agents}

Naltrexone is an opioid receptor antagonist approved by the FDA for treatment of alcohol and opioid addictions. Treatment of NSSI with naltrexone is based on phenomenologic similarities of NSSI and addiction. Indeed, it has been hypothesized that the response to naltrexone may be predicted by elevated beta-endorphin levels following self-injurious behavior. Although naltrexone is the most studied pharmacotherapy for NSSI, 
Standard dosing

Contraindications

Main side effects

Special points

Cost little consensus exists as to its efficacy due to methodological inconsistencies and study design limitations. For example, a recent review of four randomized control trials (described further in the text) comparing the effects of naltrexone versus placebo in 42 adults with intellectual disabilities was unable to reach any definite conclusions regarding efficacy due to low power [30•]. The first of these trials reported that naltrexone attenuated SIB in all four participants, with $25 \mathrm{mg}$ and $50 \mathrm{mg}$ doses producing a statistically significant decrease in SIB [31]. The second trial included eight patients and results indicated that naltrexone was associated with significantly fewer days of high frequency self-injury and significantly more days with low frequency self-injury [32]. The third study of 26 participants found that neither single-dose (100 mg) nor long-term (50 and $150 \mathrm{mg}$ ) naltrexone treatment had any therapeutic effect on SIB [33]. Finally, naltrexone had clinically significant effects ( $\geq 33 \%$ reduction) on the daily rates of three of the four participants' most severe forms of SIB, but results did not reach statistical significance in the most recent study included in the review [34]. There were very few noteworthy adverse events in any of the four trials that were reviewed. All trials were at high risk of bias and the short period of follow-up was a significant drawback in their design; the authors of the review thus concluded that there was "weak evidence in included trials that naltrexone was more effective than placebo for people with intellectual disability demonstrating SIB". Of interest, Symons and colleagues conducted a meta-analysis of 27 uncontrolled studies of naltrexone in 86 patients with mental retardation and NSSI and reported that $80 \%$ of participants experienced a decrease in frequency or severity of NSSI behaviors following administration of naltrexone [35].

$50-100 \mathrm{mg} / \mathrm{d}$

Naltrexone is contraindicated in concurrent use with opioid analgesics or in patients currently dependent on opioids, including those currently maintained on opiate agonists.

Generally well-tolerated; may cause nausea, vomiting, headache, dizziness, anxiety, and fatigue.

Naltrexone can cause hepatocellular injury when given in higher doses, and it is contraindicated in acute hepatitis or liver failure.

cost-effective as it is available in generic formulation

Buprenorphine

Buprenorphine is a semi-synthetic partial opioid agonist that is used to treat opioid addiction and to control moderate acute and chronic pain. A recent small and uncontrolled study suggested successful treatment of severe NSSI with buprenorphine $(0.5-6 \mathrm{mg} / \mathrm{d})$ in six individuals [36].

\section{Monoamine-modulating agents}

The monoamine dysregulation found in NSSI prompted selective serotonin reuptake inhibitors (SSRIs) to be examined as treatments for reducing SIB, 
but no systematic research has been conducted on the benefits of antidepressants for the reduction of NSSI, and currently available data are mixed. A review of effectiveness of antidepressants in management of behavior problems, including but not limited to SIB, in adults with intellectual disabilities reported mixed results with more than $50 \%$ of cases showing either no improvement or deterioration [37]. Fluoxetine has been investigated as a treatment for pathological skin picking, a condition which is possibly related to NSSI. While two controlled studies reported significant improvement of symptoms compared to placebo [38, 39], a number of published reports failed to replicate such positive results [40, 41]. A comparison of clomipramine versus placebo in individuals with mental retardation found no statistically significant benefit for any outcome measure, including SIB rate and intensity, but clinically significant improvement in the rate and intensity of SIB was reported [42]. Available data do not support the use of antidepressants as first line of treatment for NSSI unless, perhaps, when NSSI is associated with depressive symptoms.

\section{Glutamate modulating agents}

N-acetylcysteine (NAC)

NAC is an amino acid and cysteine pro-drug which reduces synaptic release of glutamate. In the United States, NAC is available as a prescription product used in the treatment of cystic fibrosis and other chronic pulmonary conditions [43] and as an intravenous formulation used to treat acetaminophen overdose [44]. NAC is also available without prescription as an over-the-counter product in health food stores, usually promoted as improving overall mental function. Few reports have been published testing NAC for NSSI, DSH, or SIB, and results remain mixed. In a case study, Pittenger and colleagues reported decreases in self-injurious behaviors using NAC for a patient who experienced similar decreases in SIB on riluzole (another glutamate-modulating agent), but could not tolerate the sedative effect of the medication [45]. While NAC treatment produced conflicting results in trichotillomania studies [46, 47], a recent overview published by Grant and colleagues recommended NAC as a pharmacological treatment strategy for skin-picking disorder [48]. Existing data on NAC in NSSI are limited and controversial and do not support the use of NAC as first-line treatment for NSSI. Nonetheless, NAC might improve some NSSI and its beneficial side effects profile, availability, and low cost should be taken in consideration when adjunctive medication in the armamentarium of agents used for NSSI is discussed.

Topiramate is an anticonvulsant medication and while its precise mechanism of action remains unknown, blockage of voltage-dependent sodium channels and antagonism the AMPA/kainate subtype of the glutamate receptor might contribute to its clinical properties. Preliminary uncontrolled data suggested that topiramate might help decrease skin picking [49, 50], trichotillomania [51] and similar self-injurious behaviors [52], 
particularly in individuals with intellectual disabilities. Existing data on topiramate in NSSI is very limited, but similarly to NAC, it should be taken into consideration when adjunctive medication for NSSI is discussed.

\section{Compliance with Ethics Guidelines}

\section{Conflict of Interest}

Dr. Guerdjikova declares no conflict of interest

Dr. McCullumsmith is employed by the University of Cincinnati College of Medicine, and the University of Cincinnati Physicians. She is a consultant to, and has served as a member of, the scientific advisory board for Janssen Scientific in the past year.

Dr. Trisha Suppes is employed by Stanford University and Veteran Affairs Hospital in Palo Alto. In the past year, she has received research funding from the National Institute of Mental Health, Elan Pharma International Limited, and the VA Cooperative Studies Program; and has been a consultant to Inc. H. Lundbeck A/S, Merck, and AstraZeneca. Dr. McElroy is employed by the University of Cincinnati College of Medicine, University of Cincinnati Physicians, and the Lindner Center of HOPE. Dr. McElroy is a consultant to, or member of the scientific advisory boards, in the past year to Bracket; F. Hoffmann-La Roche Ltd.; MedAvante; Naurex; Shire; Sunovion. Dr. McElroy is presently, or has been in the past year, a principal or co-investigator on research studies sponsored by the Agency for Healthcare Research \& Quality (AHRQ); Alkermes; Cephalon; Forest; Marriott Foundation; National Institute of Mental Health; Naurex; Orexigen Therapeutics, Inc.; Shire ; Takeda Pharmaceutical Company Limited. Patents: Dr. Susan L. McElroy is also inventor on United States Patent No. 6,323,236 B2, Use of Sulfamate Derivatives for Treating Impulse Control Disorders, and, along with the patent's assignee, University of Cincinnati, Cincinnati, OH, has received payments from Johnson \& Johnson Pharmaceutical Research \& Development, L.L.C., which has exclusive rights under the patent.

Human and Animal Rights and Informed Consent

This article does not contain any studies with human or animal subjects performed by any of the author.

\section{References and Recommended Reading}

Papers of particular interest, published recently, have been highlighted as:

- Of importance

1. Gilman SL. How new is self-harm? J Nerv Ment Dis. 2012;200(12):1008-16.

2. Mangnall J, Yurkovich E. A literature review of deliberate self-harm. Perspect Psychiatr Care. 2008;44(3):175-84.

3. Lloyd-Richardson EE et al. Characteristics and functions of non-suicidal self-injury in a community sample of adolescents. Psychol Med. 2007;37(8):1183-92

4. Briere J, Gil E. Self-mutilation in clinical and general population samples: prevalence, correlates, and functions. Am J Orthopsychiatry. 1998;68(4):609-20.

5. Jacobson CM, Gould M. The epidemiology and phenomenology of non-suicidal self-injurious behavior among adolescents: a critical review of the literature. Arch Suicide Res. 2007;11(2):129-47.

6. Klonsky ED. Non-suicidal self-injury in United States adults: prevalence, sociodemographics, topography and functions. Psychol Med. 2011;41(9):1981-6.
This is a novel study examining lifetime and 12-month prevalence of Non-suicidal self-injury by random-digit dialing in a sample of 439 adults in the United States. Lifetime prevalence of NSSI was $5.9 \%$, and the 12 -month prevalence was $0.9 \%$. The average age of onset was 16 years of age. The study concluded that NSSI was associated with younger age, being unmarried and a history of mental health treatment, but not with gender or ethnicity.

7. Zetterqvist $\mathrm{M}$ et al. Prevalence and function of nonsuicidal self-injury (NSSI) in a community sample of adolescents, using suggested DSM-5 criteria for a potential NSSI disorder. J Abnorm Child Psychol. 2013;41(5):759-73.

8. Ferrara $M$, Terrinoni A, Williams R. Non-suicidal selfinjury (Nssi) in adolescent inpatients: assessing personality features and attitude toward death. Child Adolesc Psychiatry Ment Health. 2012;6:12.

9. Plener PL et al. An international comparison of adolescent non-suicidal self-injury (NSSI) and suicide 
attempts: Germany and the USA. Psychol Med. 2009;39(9):1549-58.

10. Gratz KL, Conrad SD, Roemer L. Risk factors for deliberate self-harm among college students. Am J Orthopsychiatry. 2002;72(1):128-40.

11. Darke S et al. Attempted suicide, self-harm, and violent victimization among regular illicit drug users. Suicide Life Threat Behav. 2010;40(6):587-96.

12. A.P.A. Diagnostic and Statistical Manual of Mental Disorders (Fifth edition). 2013.

13. Victor SE, Klonsky ED. Correlates of suicide attempts among self-injurers: A meta-analysis. Clin Psychol Rev. 2014;34(4):282-97.

This paper presented a systematic review of 52 studies examining predictors of suicide attempts (SA) history among selfinjurers. The strongest correlate of SA history was suicidal ideation, followed by NSSI frequency, number of NSSI methods, and hopelessness. Moderate predictors of SA history included Borderline Personality Disorder, impulsivity, PostTraumatic Stress Disorder, the NSSI method of cutting, and depression.

14. Nock MK et al. Self-Injurious Thoughts and Behaviors Interview: development, reliability, and validity in an adolescent sample. Psychol Assess. 2007;19(3):30917.

15. Linehan MM et al. Suicide Attempt Self-Injury Interview (SASII): development, reliability, and validity of a scale to assess suicide attempts and intentional selfinjury. Psychol Assess. 2006;18(3):303-12.

16. Martin J et al. Psychometric properties of the functions and addictive features scales of the Ottawa self-injury inventory: a preliminary investigation using a university sample. Psychol Assess. 2013;25(3):1013-8.

17. Tarrier N, Taylor K, Gooding P. Cognitive-behavioral interventions to reduce suicide behavior: a systematic review and meta-analysis. Behav Modif.

2008;32(1):77-108.

18. Slee $\mathrm{N}$ et al. Cognitive-behavioral therapy for deliberate self-harm. Crisis. 2007;28(4):175-82.

19. Evans K et al. Manual-assisted cognitive-behaviour therapy (MACT): a randomized controlled trial of a brief intervention with bibliotherapy in the treatment of recurrent deliberate self-harm. Psychol Med. 1999;29(1):19-25.

20. Tyrer P et al. Randomized controlled trial of brief cognitive behaviour therapy versus treatment as usual in recurrent deliberate self-harm: the POPMACT study. Psychol Med. 2003;33(6):969-76.

21. Taylor LM et al. Development and pilot evaluation of a manualized cognitive-behavioural treatment package for adolescent self-harm. Behav Cogn Psychother. 2011;39(5):619-25.

22. Stanley B et al. Brief dialectical behavior therapy (DBTB) for suicidal behavior and non-suicidal self injury. Arch Suicide Res. 2007;11(4):337-41.

23. Kliem S, Kroger C, Kosfelder J. Dialectical behavior therapy for borderline personality disorder: a metaanalysis using mixed-effects modeling. J Consult Clin Psychol. 2010;78(6):936-51.
24. Fleischhaker C et al. Dialectical Behavioral Therapy for Adolescents (DBT-A): a clinical Trial for Patients with suicidal and self-injurious Behavior and Borderline Symptoms with a one-year Follow-up. Child Adolesc Psychiatry Ment Health. 2011;5(1):3.

25. Bateman A, Fonagy P. 8-year follow-up of patients treated for borderline personality disorder: mentalization-based treatment versus treatment as usual. Am J Psychiatry. 2008;165(5):631-8.

26. Rossouw TI, Fonagy P. Mentalization-based treatment for self-harm in adolescents: a randomized controlled trial. J Am Acad Child Adolesc Psychiatry. 2012;51(12):1304-1313 e3.

27. Hatcher S et al. Problem-solving therapy for people who present to hospital with self-harm: Zelen randomised controlled trial. Br J Psychiatry. 2011;199(4):310-6.

28. Inckle K. At the cutting edge: creative and holistic responses to self-injury. Creat Nurs. 2010;16(4):160-5.

29. Wallenstein MB, Nock MK. Physical exercise as a treatment for non-suicidal self-injury: evidence from a single-case study. Am J Psychiatry. 2007;164(2):350-1.

30. Rana F, Gormez A, Varghese S. Pharmacological interventions for self-injurious behaviour in adults with intellectual disabilities. Cochrane Database Syst Rev. 2013;4:CD009084.

This study is the most current and comprehensive review of controlled pharmacotherapy trials in NSSI to date. The review included five double-blind placebo-controlled trials and assessed effectiveness and safety of naltrexone and clomipramine in a total of 50 people with intellectual disabilities demonstrating SIB. The study concluded that there was weak evidence that any active drug was more effective than placebo.

31. Sandman CA, Barron JL, Colman H. An orally administered opiate blocker, naltrexone, attenuates selfinjurious behavior. Am J Ment Retard. 1990;95(1):93102.

32. Thompson T et al. Opioid antagonist effects on selfinjury in adults with mental retardation: response form and location as determinants of medication effects. Am J Ment Retard. 1994;99(1):85-102.

33. Willemsen-Swinkels $\mathrm{SH}$ et al. Failure of naltrexone hydrochloride to reduce self-injurious and autistic behavior in mentally retarded adults. Double-blind placebo-controlled studies. Arch Gen Psychiatry. 1995;52(9):766-73.

34. Symons FJ et al. Sequential analysis of the effects of naltrexone on the environmental mediation of selfinjurious behavior. Exp Clin Psychopharmacol. 2001;9(3):269-76.

35. Symons FJ, Thompson A, Rodriguez MC. Self-injurious behavior and the efficacy of naltrexone treatment: a quantitative synthesis. Ment Retard Dev Disabil Res Rev. 2004;10(3):193-200.

36. Norelli LJ et al. Buprenorphine in the treatment of nonsuicidal self-injury: a case series and discussion of the literature. Int J Adolesc Med Health. 2013;25(3):32330 . 
37. Sohanpal SK et al. The effectiveness of antidepressant medication in the management of behaviour problems in adults with intellectual disabilities: a systematic review. J Intellect Disabil Res. 2007;51(Pt 10):750-65.

38. Simeon D et al. A double-blind trial of fluoxetine in pathologic skin picking. J Clin Psychiatry.

1997;58(8):341-7.

39. Bloch MR et al. Fluoxetine in pathologic skin-picking: open-label and double-blind results. Psychosomatics. 2001;42(4):314-9.

40. Sharma H. Psychogenic excoriation responding to fluoxetine: a case report. J Indian Med Assoc.

2008;106(4):245-62.

41. Schepis C et al. Skin-picking: the best cutaneous feature in the recognization of Prader-Willi syndrome. Int J Dermatol. 1994;33(12):866-7.

42. Lewis $\mathrm{MH}$ et al. Clomipramine treatment for selfinjurious behavior of individuals with mental retardation: a double-blind comparison with placebo. Am J Ment Retard. 1996;100(6):654-65.

43. Stey $\mathrm{C}$ et al. The effect of oral $\mathrm{N}$-acetylcysteine in chronic bronchitis: a quantitative systematic review. Eur Respir J. 2000;16(2):253-62.

44. Donovan JW, Burkhart KK. N-acetylcysteine in treatment of acetaminophen overdose. J Clin Gastroenterol. 1992;15(2):169-70.

45. Pittenger C, Krystal JH, Coric V. Initial evidence of the beneficial effects of glutamate-modulating agents in the treatment of self-injurious behavior associated with borderline personality disorder. J Clin Psychiatry. 2005;66(11):1492-3.

46. Grant JE, Odlaug BL, Kim SW. N-acetylcysteine, a glutamate modulator, in the treatment of trichotillomania: a double-blind, placebo-controlled study. Arch Gen Psychiatry. 2009;66(7):756-63.

47. Bloch $\mathrm{MH}$ et al. N-Acetylcysteine in the treatment of pediatric trichotillomania: a randomized, doubleblind, placebo-controlled add-on trial. J Am Acad Child Adolesc Psychiatry. 2013;52(3):231-40.

48. Grant JE et al. Skin picking disorder. Am J Psychiatry. 2012;169(11):1143-9.

49. Jafferany M, Shireen F, Ibrahim A. An open-label trial of topiramate in the treatment of skin picking in pervasive developmental disorder not otherwise specified. Prim Care Companion. J Clin Psychiatry. 2010;12(2).

50. Shapira NA et al. Effects of topiramate in adults with Prader-Willi syndrome. Am J Ment Retard. 2004;109(4):301-9.

51. Lochner $\mathrm{C}$ et al. Topiramate in the treatment of trichotillomania: an open-label pilot study. Int Clin Psychopharmacol. 2006;21(5):255-9.

52. Janowsky DS et al. Effects of topiramate on aggressive, self-injurious, and disruptive/destructive behaviors in the intellectually disabled: an open-label retrospective study. J Clin Psychopharmacol. 2003;23(5):500-4. 